\title{
Considerations for Believable Emotional Facial Expression Animation
}

\author{
Robin J.S. Sloan, Malcolm Cook, Brian Robinson \\ University of Abertay Dundee \\ Dundee, United Kingdom \\ r.sloan@abertay.ac.uk,m.cook@abertay.ac.uk,b.robinson@abertay.ac.uk
}

\begin{abstract}
Facial expressions can be used to communicate emotional states through the use of universal signifiers within key regions of the face. Psychology research has identified what these signifiers are and how different combinations and variations can be interpreted. Research into expressions has informed animation practice, but as yet very little is known about the movement within and between emotional expressions. A better understanding of sequence, timing, and duration could better inform the production of believable animation. This paper introduces the idea of expression choreography, and how tests of observer perception might enhance our understanding of moving emotional expressions.
\end{abstract}

Keywords - Character Animation, Facial Animation, Emotional Expression, Believability, Perception

\section{INTRODUCTION}

In the digital domain, there is clear opportunity to promote lifelike behaviour in computer-based characters. Believable acting from game characters, for instance, can enhance the game play and narrative immersion of computer games, educational tools, and training simulators.

Motion capture has been a popular method of producing realistic animation for games and film. Several high profile films have incorporated performance capture techniques [8, 29]. Increasingly, performance capture is being utilized in games as well. For example, Image Metrics [19] provide capture services using video of actors, creating believable animation for games as well as for film.

However, motion capture does little to address the needs of fully interactive characters. In order to produce real-time animation in reaction to user input, a better understanding of human movement perception is needed.

One specific area of interest is emotional facial expression and recognition. Research into how observers perceive facial expression has informed animation training and artistic practice $[15,21]$. However, not much is known about the intricacies of expression dynamics, in particular as regards emotional expression choreography - the sequence, timing, and duration of facial movements.

Investigation into audience perception of variation in emotional expression choreography could lead to advanced training guides for character animation, and also lay the ground work for believable real-time animation of interactive characters.

'Emotional Avatars' is an interdisciplinary research project which looks to use psychology-based methods to inform artistic practice. The primary goal of the project is to produce and test animations of and between emotional expressions, with variance in feature sequence, timing, and duration. This paper will cover initial facial expression perception research and sequence manipulation.

\section{A. Related research}

There have been a number of studies into the generation and perception of emotional facial expressions, primarily in psychology and computing research.

While research into facial expression observation can be dated back to Darwin [9], most current studies into facial expressions consider the Facial Action Coding System (FACS) developed by Ekman and Friesen [11] to be the first major step towards a scientific understanding of facial expressions. By breaking expressions down into Action Units, FACS has not only informed subsequent studies into observation, but has also informed animation practice and computing projects - for example the development of MPEG-4 standard [6], the Maya-based Facial Animation Toolset [20], and iFACE [3].

Further psychological studies into facial perception have helped to identify universal expressions [10]. These expressions - most often cited as happiness, sadness, anger, fear, disgust, and surprise - are considered universal in the sense that the majority of observers, regardless of cultural background, can accurately identify the correct emotion.

However, many studies into facial perception focus on the use of still photographs, drawings, or static computer generated images. One of the first studies into facial movement perception was by Bassili [5], who found that universal emotional expressions could be recognized by subjects when only the motion on the surface of the face was observed. Further studies have shown that dynamic presentation of expressions can enhance recognition accuracy $[2,37]$ which can perhaps be explained by the theory that static and dynamic facial expressions prompt different brain processes [1]. This would suggest that - while static images are useful as a guide - only research into dynamic presentation and perception of expressions can truly inform animation practice.

Blending (morphing) between two established expressions is a common approach for animation generation in both computing [22, 31] and psychology [26] research. This can be an effective way to simplify the transition between states, but, even when more consideration is given to how the morph is processed, blends do not necessarily 
consider how temporal subtleties can affect expression recognition or authenticity.

It has been shown that certain expressions might have typical movements in terms of the sequence of facial regions. For instance, smiles could start with lower face movement (the smile) and finish with upper face movement (squinting around the eyes, known as the Duchenne smile) [25]. However, the temporal unfolding of facial regions has not been tested thoroughly with the universal expressions, and the effect of sequence manipulation on perceived believability has not been investigated

Nuanced sequential movements made up of distinct facial actions over time (known as appraisal theory) have also been suggested [37], although the effect on observer perception is inconclusive. Studies into appraisal theory using computer generated faces may yet produce evidence that specific sequences of movement appear more authentic than simultaneous movement [23]. However, by using such specific sequences of facial actions, it may prove difficult to conclusively show that one theorized sequence is more authentic than all other potential sequences.

The importance of facial regions to emotional states has been examined. For example, Griesser et al [17] animated the facial regions of characters to test observer perception of emotion. Nusseck et al [27] manipulated the presence of facial regions to investigate their relative contribution to expression recognition.

By showing that Action Units can be attributed to emotional dimensions, Arya et al [4] demonstrated how perceptually valid mixed emotions can be produced, which could lay the groundwork for authentic emotional transitions. However, their research focuses on the significance of features to emotional states, and does not consider the effect of temporal subtleties.

Wallraven et al [36] investigated the spatiotemporal characteristics of expressions, in essence manipulating the appearance of facial features over time. Their study looked at the effects of shape, texture, and temporal manipulation, but did not look specifically at sequence and timing issues.

One further consideration - which is especially critical to animation research - is the believability of expressions. Most guides to or papers on character animation discuss the importance of believability, using synonyms such as lifelike, sincerity, or authenticity [7, 18, 30, 33]. However, not all psychological studies into facial perception consider this factor, focusing instead on emotion identification rates, intensity, and typicality. Wallraven et al [35] took three measurements for emotional perception; identification, intensity and sincerity. Their study recognized the fact that identification of emotion is not sufficient, since observer belief in that emotional expression might still be weak.

\section{FIRST EXPERIMENT}

Before moving on to examine an aspect of choreography, an experiment was planned that would test the perceptual validity of original animated expressions. These animations were produced iteratively by the authors, drawing on Ekman's work [10] and building on research into naturalistic expression perception [34].

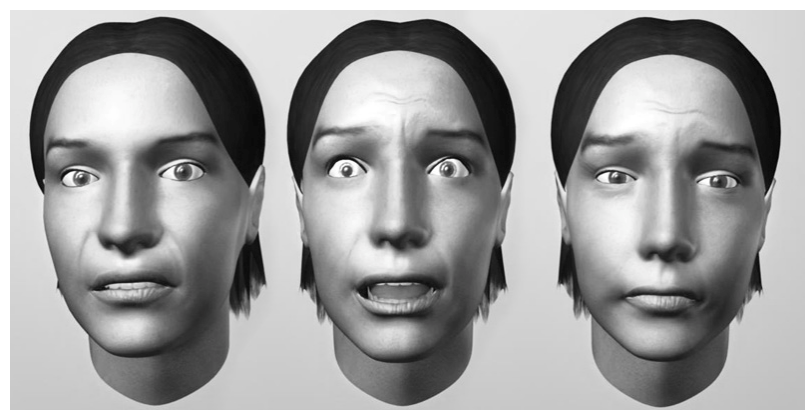

Figure 1. 3D head rendered in Maya 7.0. Expressions shown from left to right are; disgust, fear, and sadness

Ultimately, this experiment would set a benchmark for observer rating of authentic expressions, but the data would also be interrogated to determine the relationships between observer confidence of identification, intensity of emotion, and authenticity of expression.

\section{A. Animation preparation}

The animation of facial expressions was produced using Maya 7.0 [24]. A number of approaches and additional toolsets were considered - for instance the joint-based Facial Animation Toolset [20]. However, for the purposes of this experiment, the standard Maya BlendShapes tool in combination with appropriate plug-ins [28] proved to be the most adaptable and customizable approach to animation.

While this approach would limit the animation to a single head model, it was judged that variation in visual appearance would not be a critical aspect of the current research. Features such as skin colour, skin texture, eye colour, facial hair, hair style, and hair colour can be easily manipulated using this animation setup. The only aspect of the head that cannot be changed easily is the physical structure of the head, for instance the size of the jaw, length of the nose, or spacing between the eyes. This makes it difficult to vary the masculinity or femininity of the head. As such, it was decided that the computer generated head should be neither strongly masculine nor feminine.

Building a physically accurate 3D computer model of a human head is a complicated and time consuming process. One particular problem can be the surface structure of the head, which - if constructed incorrectly - can cause issues during animation.

To save time and generate a robust 3D model, FaceGen Modeller [14] was employed. FaceGen has been used for the production of realistic computer generated heads on a number of high profile computer games [13, 16]. The software has also been used in research [32]. As such, the visual appearance and technical quality of FaceGen models have been scrutinized extensively, both commercially and academically. Fig. 1 depicts the finished head model.

\section{B. Stimuli}

Eighteen animations were produced using the facial animation setup described in section II $A$. The animations were of six emotions (happiness, sadness, anger, fear, disgust, and surprise) at three levels of emotional intensity (low, moderate, and high). Animations lasted between two 
and twenty seconds, and consisted of an onset, peak expression, and offset.

Information on muscle movement and strength was derived from FACS [11] and - in particular - from Unmasking the face [10], which describes in detail the various shapes and movements associated with particular emotional expressions. Existing images and videos of expressions [20] were used as references. Further references for movement, strength and timing were drawn from the acted and naturalistic videos described in Sloan et al [34]. The naturalistic videos had been tested on observers using a method similar to the one employed for this experiment.

Animation was produced iteratively by the authors. The resulting videos were examined by the team to identify any potential problems with the movement, isolating facial regions and points in time. Three iterations were required to produce videos deemed acceptable by the team. The final eighteen animations were then used as the stimuli for the experiment.

\section{Method}

The purpose of the experiment was to test observer perception of individual dynamic emotional expressions based on four factors; identification of emotion, confidence of identification, intensity of emotion, and authenticity of expression. Participants watched the eighteen animations and answered the following questions; Q1. which of the following emotions were expressed? (choosing one or multiple from happiness, sadness, anger, fear, disgust, surprise, and other), Q2. how confident are you that this emotion was expressed? (5-point scale from not confident to very confident), Q3. how intense was this emotion? (5-point scale from low intensity to high intensity), and Q4. how authentic was this emotional expression? (5-point scale from fake to genuine).

To display the videos and collect observer input, a computer program was developed. Videos were randomized for each participant and displayed at a resolution of 720x576 pixels, centered on a $1920 \times 1200$ screen. Participants sat in isolation for the duration of the experiment, and answered the questions after each video. The experiment generally lasted no longer than thirty minutes.

Thirty-six participants (twenty female and sixteen male) were tested.

\section{Results}

All eighteen animations were identified by observers as the correct emotion above chance. Chance rates were set at levels suggested in Ekman [12]: happiness (50\%), surprise (33\%), and sadness, anger, fear, and disgust (all 25\%).

Surprise received the highest identification rates (accuracy percentages of $97.1 \%$ for low intensity, 100\% for moderate, $97.2 \%$ for high) with no other emotion identified above chance. Other well identified expressions were happiness $(86.1 \%, 91.7 \%, 80.6 \%)$, sadness $(76.5 \%, 88.9 \%$, $97.2 \%)$, disgust $(88.6 \%, 91.7 \%, 97.2 \%)$, and anger $(64.7 \%$, $73.5 \%, 86.1 \%$ ). For animations of surprise, happiness, sadness, and disgust, no other emotion was identified above chance. For two animations of anger, disgust was identified just above chance (26.5\% in moderate intensity anger, $27.8 \%$ in high intensity anger).

Fear was the only expression observers had some difficulty with. While they identified it as fear $(47.2 \%, 75 \%$, $80 \%)$ they also identified it as surprise above chance $(55.6 \%$, $38.9 \%, 41.7 \%$ ).

In terms of intensity rating, the median value of observer ratings would suggest that, generally, observers were able to rank expressions accordingly. The following values correspond to the median ratings of low, moderate, and high intensity expressions respectively; surprise $(2,3.5,5)$, happiness $(1.5,2,3)$, sadness $(2,4,4.5)$, disgust $(2,4,5)$, and anger $(2,3,4)$. Again, fear was difficult for observers to rate $(0,3,3)$.

To investigate the association between dependent variables, the Spearman's rank correlation test was selected. In all cases, the correlation between confidence of identification and intensity was significant: happiness $(\rho=$ $.264, p<.008)$, sadness $(\rho=.449, p<.001)$, anger $(\rho=.241$, $\mathrm{p}<.019)$, fear $(\rho=.507, \mathrm{p}<.001)$, disgust $(\rho=.510, \mathrm{p}<$ $.001)$, surprise $(\rho=.527, p<.001)$. This would indicate that perceived intensity of emotion positively co-varies with observer confidence when identifying an emotion.

A similar conclusion could be drawn regarding the association between observer confidence and perceived authenticity. In this instance, the results were as follows: happiness $(\rho=.482, p<.001)$, sadness $(\rho=.341, p<.001)$, anger $(\rho=.443, p<.001)$, fear $(\rho=.530, p<.001)$, disgust $(\rho=.487, p<.001)$, surprise $(\rho=.393, p<.001)$. As such, observers had more confidence identifying an emotion when they perceived the expression to be authentic.

The correlation between perceived intensity and perceived authenticity was not as strong. Generally, the correlation was seen to be positive yet weak, and in one cases the relationship was non-significant: happiness $(\rho=$ $.211, \mathrm{p}<.033)$, sadness $(\rho=.290, \mathrm{p}<.002)$, anger $(\rho=.170$, $\mathrm{p}<.1)$, fear $(\rho=.398, \mathrm{p}<.001)$, disgust $(\rho=.330, \mathrm{p}<.001)$, surprise $(\rho=.307, p<.001)$. This would suggest that, in most cases, expressions that were perceived to be more intense were also perceived to be more authentic. However, the correlation between these two variables was generally not as strong as the correlation between confidence and intensity or confidence and authenticity.

\section{SECOND EXPERIMENT}

The second experiment is the first in a series looking to test the theory of emotional expression choreography. At this stage, the first aspect of choreography to be investigated is the sequence of movement (see Fig. 2).

Three key regions of the face - the brows, the eyes, and the lower face - were manipulated to create a range of animated sequences for individual emotional expressions. Through testing of observer perception, the goal was to identify one or a range of sequences that enhance recognition and improve perceived authenticity of emotional expressions. At the same time, sequences which make expressions difficult to read or decrease authenticity could also be identified. 


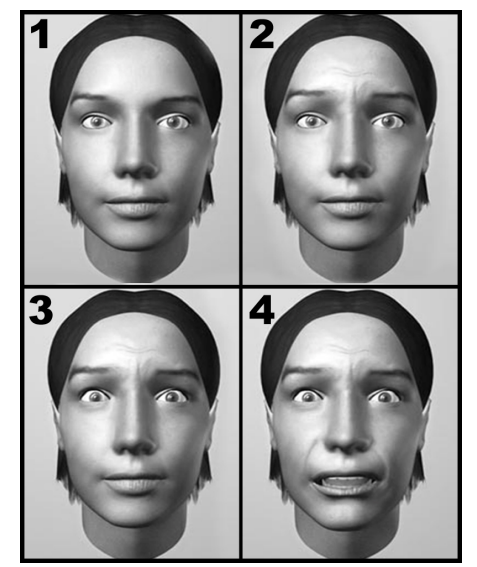

Figure 2. Sequence 1 of 'fear', starting with a neutral face (1) before moving through the brows (2), then eyes (3), then the lower face (4)

\section{A. Stimuli}

The facial rig described in section II $A$ was used to create seventy-eight animations. These 78 animations were based on three emotions (sadness, fear, and disgust) at one level of emotional intensity (high) using thirteen sequences of movement (see Table 1). The onset and offset were also separated, so that they could be observed in isolation.

The animations stemmed from those tested in the first experiment. Animated features within the brows, eyes, and lower face regions were replicated in new animations (e.g. for disgust animations a sneer was created in the lower face, and for sadness animations a raised squeeze was created in the brow region). Head movement and gaze were minimized, so that the experiment could focus on facial feature sequences only.

Sadness and disgust were selected due to high identification rates and perceived authenticity. Fear, as the most difficult emotion to recognize in the previous study, was also chosen to see if sequence manipulation could help improve recognition.

Happiness, anger, and surprise were excluded at this stage in order to minimize the number of trials.

TABLE I. THIRTEEN VARIATIONS ON EXPRESSION SEQUENCE

\begin{tabular}{|l|l|l|l|}
\hline Seq. & First & Second & Third \\
\hline 1 & Brows & Eyes & Lower \\
2 & Brows & Lower & Eyes \\
3 & Eyes & Brows & Lower \\
4 & Eyes & Lower & Brows \\
5 & Lower & Brows & Eyes \\
6 & Lower & Eyes & Brows \\
7 & Brows + Eyes & Lower & n/a \\
8 & Brows + Lower & Eyes & n/a \\
9 & Eyes + Lower & Brows & n/a \\
10 & Brows & Eyes + Lower & n/a \\
11 & Eyes & Brows + Lower & n/a \\
12 & Lower & Brows + Eyes & n/a \\
13 & Brows + Eyes + Lower & n/a & n/a \\
\hline
\end{tabular}

\section{B. Method}

Like the previous experiment, the purpose of the second experiment was to test observer perception of dynamic emotional expressions. As such, the computer program described in section II was used again to display videos and collect data. The same measures were also taken; identification of emotion, confidence of identification, intensity of emotion, and authenticity of expression.

All seventy-eight animations were shown to thirty-two subjects (sixteen female, sixteen male). To minimize the effects of participant fatigue, the experiment was split into two parts; onset animations, and offset animations. Sixteen participants watched the thirty-nine onset animations followed by the thirty-nine offset animations, while sixteen watched the thirty-nine offset animations followed by the thirty-nine onset animations. All animations were displayed in a randomized order for each participant.

\section{Results}

In terms of confidence of identification and perceived authenticity, analysis of variance (Friedman ANOVA) showed that there was no significant variation in observer ratings. However, while the results were inconclusive, the data indicates that the importance of sequence to perception could vary with the type of emotion. For instance, observers appeared to find most sequence variations acceptable for expressions of sadness, but fewer sequence variations received high ratings for fear. The control sequence of simultaneous movement (sequence 13) also tended to receive high ratings for both confidence (con) and authenticity (aut).

In most cases, observers rated the disgust animations as being primarily anger. This is interesting, since the earlier study showed that the first set of disgust animations were easily recognized as the correct emotion. One possible problem might be the strength of specific facial actions in the brow and eye regions. If these regions were weakened, the confusion with anger might be lessened (an experiment testing for this is currently under way). Accuracy of identification rates of disgust (first percentage) and anger (second percentage) for disgust onset sequences were as follows: $1(43.8 \%, 78.1 \%), 2(54.8 \%, 74.2 \%), 3(58.1 \%$, $71 \%), 4(67.8 \%, 64.5 \%), 5(58.1 \%, 74.2 \%), 6(53.1 \%, 75 \%)$, $7(61.3 \%, 61.3 \%), 8(59.4 \%, 68.8 \%), 9(56.3 \%, 68.8 \%), 10$ $(53.1 \%, 65.6 \%), 11(50 \%, 71.9 \%), 12(65.6 \%, 71.9 \%), 13$ $(64.5 \%, 58.1 \%)$. Accuracy of identification rates of disgust and anger for disgust offset sequences were: $1(67.7 \%$, $67.7 \%), 2(59.4 \%, 59.4 \%), 3(58.1 \%, 67.7 \%), 4(56.3 \%$, $68.8 \%), 5(56.3 \%, 71.9 \%), 6(45.2 \%, 74.2 \%), 7(63.3 \%$, $56.7 \%), 8(48.4 \%, 71 \%), 9(61.3 \%, 61.3 \%), 10(65.6 \%$, $59.4 \%), 11(59.4 \%, 59.4 \%), 12(56.3 \%, 68.8 \%), 13(50 \%$, $62.5 \%)$.

A few of the disgust animations received median scores of 3 or above for confidence of identification of disgust (sequences 9, 4, 5, 7, 13 for onset, 1, 2, 3, 9, 10 for offset). Across the board, the disgust sequences generally received moderate to poor scores for authenticity - likely an issue with feature strength.

Most of the fear animations were accurately and confidently identified as fear by participants, but variation in 
perceived authenticity was observed. As with the first experiment, surprise was also identified at above chance rates, but fear received higher identification rates for most sequences. Accuracy of identification rates for fear onset sequences were as follows: $1(61.3 \%), 2(77.4 \%), 3(71 \%)$, $4(78.1 \%), 5(81.3 \%), 6(75 \%), 7(78.1 \%), 8(84.4 \%), 9$ (81.3\%), $10(81.3 \%), 11(81.3 \%), 12(77.4 \%), 13(75 \%)$. Accuracy of identification rates for fear offset sequences were: 1 (78.1\%), 2 (71\%), $3(54.8 \%), 4(78.1 \%), \quad 5$ $(72.4 \%), \quad 6(71 \%), \quad 7(70 \%), \quad 8(67.7 \%), 9(78.1 \%), 10$ (62.5\%), $11(68.8 \%), 12(68.8 \%), 13(71.9 \%)$.

For onset, sequences $3,4,7,10$, and 13 received high median scores for fear $(\operatorname{con}=4$, aut $=4)$. For offset, sequences $4(\operatorname{con}=4$, aut $=4), 1$ and $12(\operatorname{con}=4$, aut $=3.5)$ received positive scores. The lowest rated sequence for onset was $6(\operatorname{con}=4$, aut $=2.5)$. For offset, the lowest rated were 3 $(\operatorname{con}=3$, aut $=2)$ and $10(\operatorname{con}=3.5$, aut $=2)$. This would suggest that - for fear - different sequences of movement might appear more or less authentic for the onset and offset of the expression. However, further research will be required to test for the significance of this variation.

Generally, sadness animations were easily identified and received medium to high median scores for both confidence of identification and authenticity. Accuracy of identification rates for sadness onset sequences were as follows: $1(100 \%)$, $2(93.8 \%), 3(96.9 \%), 4(90.6 \%), 5(93.3 \%), 6(90.6 \%), 7$ (93.8\%), $8(100 \%), 9(93.8 \%), 10(93.8 \%), 11(93.5 \%)$, $12(90.3 \%), 13(93.5 \%)$. Accuracy of identification rates for sadness offset sequences were: $1(96.8 \%), 2(93.5 \%), 3$ (90.3\%), $4(96.8 \%), 5(96.8 \%), 6(90.3 \%), 7(93.3 \%), 8$ (93.8\%), $9(96.6 \%), 10(96.9 \%), 11(84.4 \%), 12(96.9 \%)$, $13(93.5 \%)$.

For onset, only sequences $6(\operatorname{con}=5$, aut $=3), 7(\operatorname{con}=4$, aut $=3.5)$, and $9(\operatorname{con}=5$, aut $=3.5)$ received ratings below 4. Even then, the scores were still relatively high in comparison with the other emotions. For offset, every sequence received a median of 4 or higher for both confidence of identification and authenticity. As such, no sequence seemed to be clearly better or worse than the rest for this emotion, suggesting that sequence of movement does not have as much of an effect on the perception of sadness.

\section{DISCUSSION AND FURTHER RESEARCH}

While the second experiment produced inconclusive results, it is possible that sequence of regional facial movement may have an effect on how observers perceive certain emotional expressions. However, more research is required in order to understand clearly how emotional expression choreography affects perception.

By looking at all potential regional sequences in all configurations, thirteen conditions were used in the second experiment. The large number of conditions may have had an effect on observer ratings, making it difficult to clearly distinguish which sequences observers perceived to be more or less authentic. However, the control sequence of simultaneous regional movement received the highest scores for authenticity and observer confidence in a number of cases. This might indicate that sequential movement does hamper observer perception under certain conditions, and that, generally, simultaneous movement may appear more authentic to audiences.

To investigate this, the next experiment will include animations that depict three (instead of thirteen) variations on sequence. The brows and eyes will be treated as one facial region (upper face), creating just three sequence conditions; simultaneous regional movement, upper face into lower face movement, and lower face into upper face movement. All six universal expressions will also be tested. By minimizing the number of conditions, it should be possible to demonstrate the effect of regional sequence manipulation on perceived authenticity. One prediction could be that lower face into upper face movement should appear more authentic than other sequences for happiness, in line with the natural appearance of smiles [25].

Another prediction, based on observed footage of actors [34], is that sequence might play a more important role between emotions. The experiments discussed in this paper consisted of animations of single emotional expressions. An upcoming experiment will instead manipulate the sequence of regional facial movement for expression transitions, for example the facial movement between an expression of happiness and an expression of sadness.

Feature strength was discussed as one of the potential reasons why animations of disgust were confused with anger (i.e. brow and eye motion was too strong relative to the lower face, suggesting an angry upper face rather than a disgusted one). However, the first experiment included animations of disgust which had similar facial features and feature strengths to the animations used in the second experiment. Animations of disgust were clearly identified as disgust in the first experiment, but confused with anger in the second experiment. Since the first batch of animation was artistically crafted while the second was focused on the systematic manipulation of sequence, could it be that the nuances of animated performance go a long way towards communicating emotional states? For instance, the original disgust animations consisted of subtle movements, which while based on psychology research - were crafted artistically and perfected through subjective observation and reflective practice. It could be that head motion, gaze, tongue movement, or asymmetrical features enhance and clarify recognition of emotions, and that knowledge of facial feature sequence alone is not sufficient for authentic expression animation.

To investigate this, an animation project involving a group of undergraduate computer art students is being planned. By comparing emotional expression animation generated by a number of animators, subtler facial cues might be identified that - at least in the animators mind - are key to the effective expression of emotions.

\section{SUMMARY}

This paper has introduced the theory of emotional expression choreography, which can be described as the sequence, timing, and duration of facial features within and between moving expressions. Two experiments were explained, both of which looked at observer perception of animated emotional expressions. 
The first experiment demonstrated that observers can discriminate between original animated emotional expressions, and that relationships exist between observer confidence in identification of emotion, perceived intensity of emotion, and perceived authenticity of expression.

The second experiment looked into variation in sequence - the first aspect of choreography. Differences in observer ratings of authenticity were non-significant, but the results indicate that further research is needed to determine the true effect of regional sequence on observer perception.

Building on these experiments, the next steps will be to look more closely at sequence as well as other aspects of expression choreography, and to study how manipulation of choreography affects perception. Further research will investigate whether perceptually valid and invalid choreographies (based on sequence, timing and duration) can be identified for particular emotional expressions and emotional expression transitions. In turn, documentation of potential valid and invalid choreographies will be used as a means to inform animation practice.

\section{REFERENCES}

[1] Adolphs, R. (2002). 'Recognizing emotion from facial expressions: psychological and neurological mechanisms'. Behavioral and Cognitive Neuroscience Reviews. 1(1): 21-62.

[2] Ambadar, Z., Schooler, J.W., Cohn, J.F. (2005). 'Deciphering the enigmatic face: The importance of facial dynamics in interpreting subtle facial expressions'. Psychological Science. 16(5): 403-410.

[3] Arya, Ali., and DiPaola, Steve. (2006), 'Socially communicative characters for interactive applications'. The 14th International Conference in Central Europe on Computer Graphics, Visualization and Computer Vision, Plzen, Czech Republic

[4] Arya, A., DiPaola, S., Parush, A. (2009). 'Perceptually valid facial expressions for character-based applications'. International Journal of Computer Game Technology. (Accepted for publication 2009).

[5] Bassili, J.N. (1979). 'Emotion recognition: The role of facial movement and the relative importance of upper and lower areas of the face'. Journal of Personality and Social Psychology, 37(11), 20492059.

[6] Battista, S., Casalino, F., Lande, Claudio. (1999). 'MPEG-4: a multimedia standard for the third millennium, part 1'. IEEE MultiMedia. 6(4): 74-83. IEEE Press, 1999.

[7] Bates, J. (1994), 'The role of emotion in believable agents'. Communications of the ACM, 37(7), 122-125.

[8] Beowulf. (2007). [Film]. Directed by Robert Zemeckis. USA: ImageMovers

[9] Darwin, C. (1872), The expression of emotions in men and animals. London: John Murray.

[10] Ekman, P., and Friesen, W.V. (1975), Unmasking the face. New Jersey: Prentice-Hall

[11] Ekman P., and Friesen, W.V. (1977). Manual for the facial action coding system. Palo Alto: Consulting Psychologists Press

[12] Ekman, P. (1994). Strong evidence for universals in facial expressions: A reply to Russell's mistaken critique. Psychological Bulletin, 115, 268-287.

[13] Elder scrolls IV: oblivion. (2006). [DISC]. Xbox 360. Bethesda

[14] FaceGen Modeller 3.2 (2007). [Computer Software]. Singular Inversions.

[15] Faigin, G. (1990). The artist's complete guide to facial expression. USA: Watson-Guptil Publications.

[16] Fallout 3 (2008). [DISC]. Xbox 360. Bethesda.
[17] Griesser, R.T., Cunningham, D.W., Wallraven, C., and Bulthoff, H.H (2007). 'Psychological investigation of facial expressions using computer animated faces'. Symposium on Applied Perception in Graphics and Visualisation, Tubingen, Germany: ACM Press.

[18] Hooks, E. (2000). Acting for animators: a complete guide to performance animation. Portsmouth, NH: Heinemann.

[19] Image Metrics. (2008). Superior facial animation. Simplified. [Online]. Available at: http://www.image-metrics.com. [Accessed February $12^{\text {th }} 2009$ ].

[20] Institute of Animation, Visual Effects and Digital Postproduction. (2006). Research \& development at the Institute of Animation. [Online]. Available at: http://research.animationinstitut.de/ [accessed 10 Dec 2007]

[21] Kalwick, D.J. (2006). Animating facial features and expressions. Boston: Charles River Media.

[22] Lin, D.T., and Huang, H. (1999). 'Facial expression morphing and animation with local warping methods'. In Proceedings of the $10^{\text {th }}$ international Conference on Image Analysis and Processing, September 27-29, 1999.

[23] Malatesta, L., Raouzaiou, A., Karpouzis, K., and Kollias, S. (2009). MPEG-4 facial expression synthesis. Personal Ubiquitous Comput. 13, 1 (Jan. 2009), 77-83

[24] Maya 7.0 (2005). [Computer Software]. Alias.

[25] Messinger, D. S., Fogel, A., \& Dickson, K. L. (1999). What's in a smile? Developmental Psychology, 35, 701-708

[26] Montagne, B., Kessels, R.P.C., Frigerio, E., de Haan, E.H.F., Perret, D.I. (2005). 'Sex differences in the perception of affective facial expressions: do men really lack emotional sensitivity?'. Cognitive Processing. 6(2): 136-141.

[27] Nusseck, M., Cunningham, D. W., Wallraven, C., \& Bülthoff, H. H. (2008). 'The contribution of different facial regions to the recognition of conversational expressions'. Journal of Vision, 8(8):1, 1-23

[28] Osipa, J. (2007). Stop staring: facial modeling and animation done right. $2^{\text {nd }}$ ed. Indianapolis, Indiana: Wiley Publishing, Inc.

[29] The Polar Expess. (2004). [Film]. Directed by Robert Zemeckis. USA: Castle Rock Entertainment.

[30] Porter, T. (1997). 'Creating lifelike characters in Toy Story'. ACM SIGART Bulletin, 8(1-4), 10-14.

[31] Pyun, H., Kim, Y., Chae, W., Kang, H.W., and Shin, S.Y. (2003). 'An example-based approach for facial expression cloning'. In Proceedings of the 2003 ACM Siggraph/Eurographics Symposium on Computer Animation. San Diego, CA, Jul 26-27, 2003.

[32] Singular Inversions Inc. (2008). About singular inversions. [Online] Available at: http://www.facegen.com/ about.htm [Accessed 20th January 2009]

[33] Thomas, F., Johnston, O. (1981). The illusion of life: Disney animation. New York: Disney Editions.

[34] Sloan, R.J.S., Robinson, B., Cook, M., and Bown, J. (2008). 'Dynamic emotional expression choreography: perception of naturalistic facial expressions'. In M. Capey, B. Ip and F. Blastland, editors, SAND Conference Proceedings, Swansea, UK 24-28 November 2008. Swansea Metropolitan University: Swansea

[35] Wallraven, C., Fischer, J., Cunningham, D.W., and Bulthoff, H. (2006). 'The evaluation of stylized facial expressions'. Proceedings of the 3rd Symposium on Applied Perception in Graphics and Visualisation, New York, USA: ACM Press.

[36] Wallraven, C., Breidt, M., Cunningham, D.W., Bulthoff, H.H. (2008). 'Evaluating the perceptual realism of animated facial expressions' ACM Transactions on Applied Perception. 4(4): 1-20.

[37] Wehrle, T., Kaiser, S., Schmidt, S., and Schere, K.R. (2000). 'Studying the dynamics of emotional expression using synthesized facial muscle movements'. Journal of Personality and Social Psychology, 78(1), 105-119. 